\title{
Sunflower Mimic Robot for Development of Dual Axis Solar Tracking System
}

\author{
D. V. Nithin Kumar, VNB Prasad Sodisetty, Ch. Vasanth Kumar
}

\begin{abstract}
This Today's world depends upon utilization of some form of energy. Be it use of mobiles, vehicles, power supply in houses etc., everything functions on the basis of energy input. The use of energy derived from fossil fuels began in early 1800 and is used till date. In the current theme that calls for saving energy and reducing pollution, it's undoubtedly of great significance to make full use of solar energy.

The solar panel system sprouted with the use of a simple magnifying glass to concentrate solar energy which has now revolutionized by using a much higher solar panel system. The framework consists of webcam, electronic circuit, Microprocessor and two DC motors. This solar tracking system is autonomous, dual axis and hybrid type. This tracking system is camera-based and can track the sun continuously. By using Region of Interest algorithm, we can get the sun coordinates from the frame. These values are sent to the microcontroller to actuate the motors and reposition the panel. This framework works free of its primary settings and can be utilized in any geological area. It holds the solar panel opposite to illumination of sun to get the most extreme solar energy and hence produce most effective power yield for the duration of the day. This study yields an output of up to 2-3\% increase from a stationary solar panel.
\end{abstract}

Keywords-Solar tracking, Sunflower mimicking, Dual axis system, Maximum efficiency, Camera tracking, HSV.

\section{INTRODUCTION}

Now a day's renewable energy solutions that is collected from renewable resources are becoming popular. Among them, one of the abundant sources is solar energy. For the past years, this application is used in household, industries, schools, colleges etc. Solar system has good efficiency and maximum output. In recent days solar panels are of fixed type, which have lower efficiency than movable type. A solar tracker follower is a gadget utilized for arranging a sun powered photovoltaic board or focal point towards the sun by utilizing the sunlight based or light-based sensors associated with the machine. The advantage of solar energy is that it is unlimited and pollution-free. The only way to increase the solar energy is to arrange the panel perpendicular to the sun. Solar tracking is the best method, by following the sun and rearrange the solar panels perpendicular to solar irradiation gives higher efficiency.

M. Abdollahpoura et al. [1] in 2018, proposed "Development of a machine vision dual-axis solar tracking system", in this system a dual-axial tracker is utilized that

Revised Manuscript Received on July 10, 2019.

D. V. Nithin Kumar, Department of Mechnaical Engineering SRM Institute of Science and Technology, Chennai, T.N, India. (E-mail: nithinkumardatti@gmail.com)

VNB Prasad Sodisetty, Department of Mechnaical Engineering SRM Institute of Science and Technology, Chennai, T.N, India. (E-mail: bhavaniprasad.478@gmail.com)

Ch. Vasanth Kumar, Department of Mechnaical Engineering SRM Institute of Science and Technology, Chennai, T.N, India. (E-mail: sreevasanthkumarchoda @gmail.com) works depend on picture processing of a bar shadow. The framework was made up of a shadow casting object. The shadow of bar is captured by web cam, it is balanced in a position to carry the panel perpendicular to light to get the most extreme solar energy and thus produce the highest power output.

Francois Henaulta et al. [2] in 2018 proposed "Sun backward gazing method with multiple cameras for characteristic solar concentrators" in this paper the ideal vitality is acquired by utilizing a sun in reverse looking strategy with different cameras. This empowers quick and precise impact of sun powered concentrators in working conditions. For concentrated solar power (CSP), commissioning of optimal segment facility, monitoring damaged mirrors observation of wind loads and gravity is essential.

Yingxue Yao et al. [3] in 2014 proposed "A multipurpose dual-axis solar tracker with two tracking strategies" in this system it explains on the utilization of sun powered framework with multipurpose double pivot sunlight-based tracker. It utilizes declination clock mounting framework. This arrangement is with the typical following technique where it utilizes time-based control and sensor-based control to work in various climate conditions.

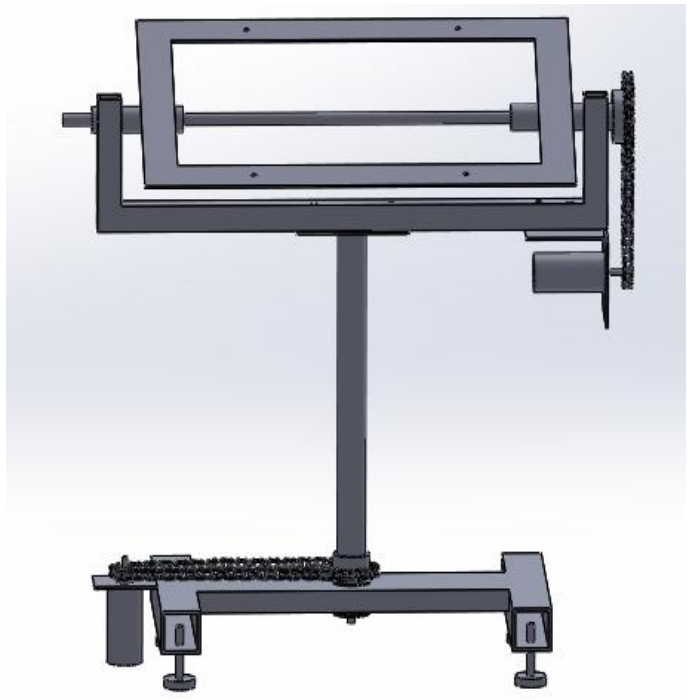

Fig. 1. Solid works Model of Frame

Tan Pau Chang [4] in 2009 proposed "Performance study on the east-west oriented single-axis tracked panel" in this theoretical study a single axis tracked panel performance in an east-west orientation was proposed. It became a

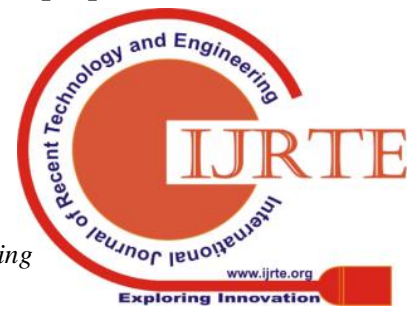


mathematical expression using which the angle of rotation of tracked panel are derived. This system gains increase with energy level of $21.2 \%, 13.5 \%$ \& $7.1 \%$ respectively low compared to that of North-South oriented single tracked panel. The irradiation received is directly proportional to latitude zone of rotating angle of the panel.

As the novelty of this paper, is to put together all these concepts and track the sun in dual axis like pan and tilt motion with single camera and controlling with DC-motor. A 3D model is designed in Solid works with required dimensions and fabricated as shown in fig. 1. In this paper the overall work process is divided in to five major segments. Section 2 describes about Tracking Methods. Section 3 gives an overview of novel method of tracking sun using HSV. Section 4 describes about controlling motion of solar panel. This paper is concluded in section 5 followed by references.

\section{TRACKING METHODS}

Based on the control unit, Sun tracking is divided into two types: passive and active system. Mostly, in passive frameworks gas liquid is utilized at low breaking point, which moves starting with one bearing then onto the next to turn the following framework. It works, in light of the varieties of gas weight caused by sun-based warmth. It is not used mostly due to its poor tracking system. This system required viscous dampers to avoid additional movement cause by air.

There are three methods of tracking based on degrees of freedom. First method is, Fixed system; in this solar panels are fixed in one direction without any movement. It does not depend in the sun's direction. Other one, Single-axis system; in this tracking system, it has one degree of freedom. Based on the suns east-west direction it moves along to that for better output. And the final one is, Dual-axis system; it is advanced and accurate tracking to increase output better than other two systems. This tracking system moves, according to the direction in east-west and north-south directions. In active system we can get more accuracy of panel and high output than passive systems.

Active systems are further divided into different types based on sensor combination and time/date. On one hand, tracking system is done by using sensor like LDR, based on the light intensity detected by the sensor the panel will move according to their degree of freedom for improvement of output energy. On other hand, the combination of sensors and time/date, in this type tracking system is done as explained above. Additional to that, it will depend on time; whether it is day or night, date; seasons like summer, winter etc. based on all these this tracking system works. The main disadvantage of this is expensive.

\section{FEATURE EXTRACTION}

In this section localizing sun is the initial thing utilized to extricate areas where sun is present. Edge characteristics are moreover considered as suitable for sun tracking. These strategies estimate centre position of sun and track it completely by looking at the dense regions in the picture. Colour edge identifiers are utilized, as the sun may also vary distinct colours like orange, yellow, red. After passing through UV filter glass, the region with white colour is considered as the sun. The tracking of sun using Open CV is shown in fig. 2 .

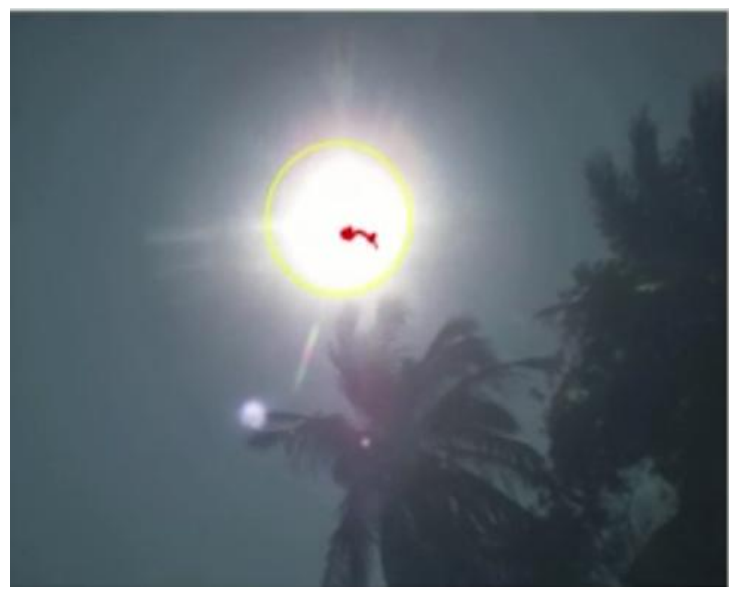

Fig. 2. Tracking Sun

\section{A. Image Acquisition}

This stage coordinates getting a picture by an acquisition method. In our proposed framework, we utilized a high or low assurance automated camera and adequate film to verify the sun from picture.

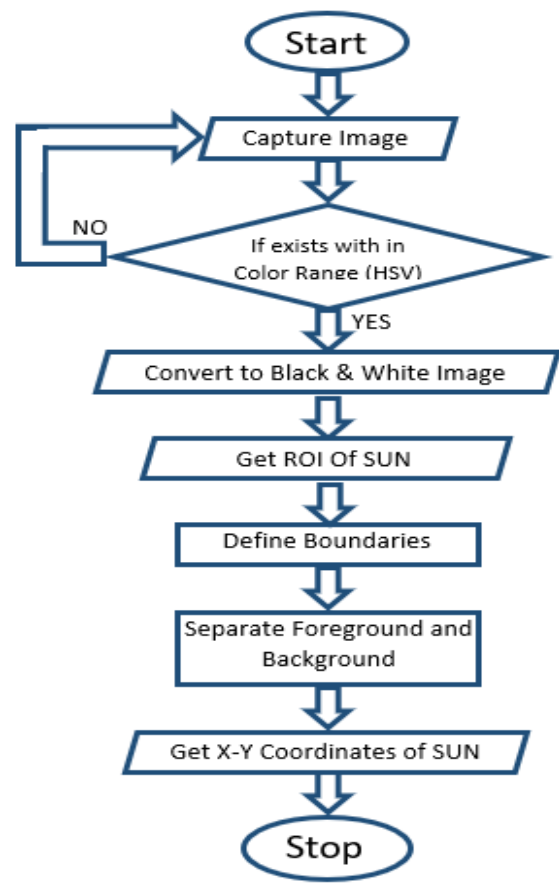

Fig. 3. Work Flow Process of Tracking Sun

\section{B. Morphological Image Processing}

The stage expels the region of interest from the captured picture. The proposed methodology first converts the image to black and white image, then get the high dense region in the picture. Mark the centre of high dense region, by applying image processing operations like erosion, dilation, etc., get the complete structure from image. Get the centre point co-ordinates.

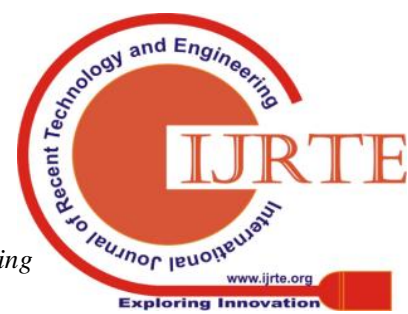




\section{HSV}

In the proposed structure HSV is utilized to localize sun. In image processing, there are various models one of which is the hue, saturation, value (HSV) model. Using this model, an object with a certain colour can be detected and to reduce the influence of light intensity from the outside.

In this system raspberry pi is utilized for processing part. Initially, when the frame is placed in the outside environment, the camera live feeds the sky. If any region in the picture exists in the given HSV range, then convert the image to black and white image. High dense and low dense regions in the image is separated. This high dense region is considered as sun. By applying image processing techniques like erosion and dilation, the complete structure is obtained i.e., boundaries of sun from picture. The centre point coordinates of sun are extracted. The work flow process of tracking is shown in fig. 3 .

\section{WORKING \& RESULTS}

In this section, the control motion of solar panel is explained. The solar panel is fixed in a such way that it should be perpendicular to the irradiation of the sun. A micro controller, motor driver, DC motors are utilized to control the motion. An accelerometer is used to define how much turn should take place for the deviation of solar panel from its required position.

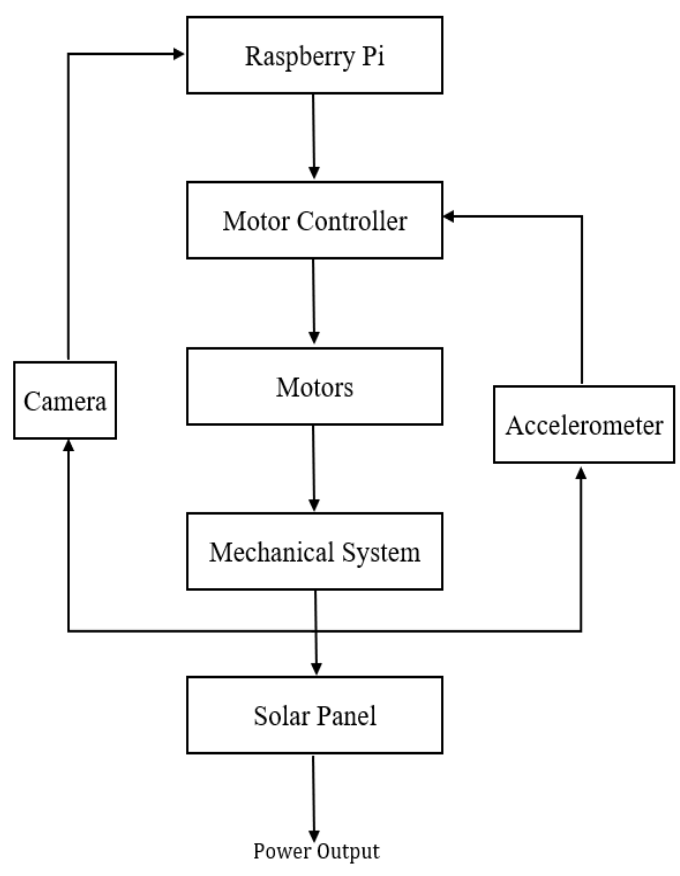

Fig. 4. Block Diagram

As shown in fig. 4 Raspberry $\mathrm{Pi}$ is utilized as main processing unit. Initially webcam is interfaced with Raspberry Pi and fixed on the solar panel to track the sun. Get the centre point coordinates of the sun, using microcontroller run the motors to move the solar panel in a required position. An accelerometer is utilized to get the angle of rotation of the solar panel.

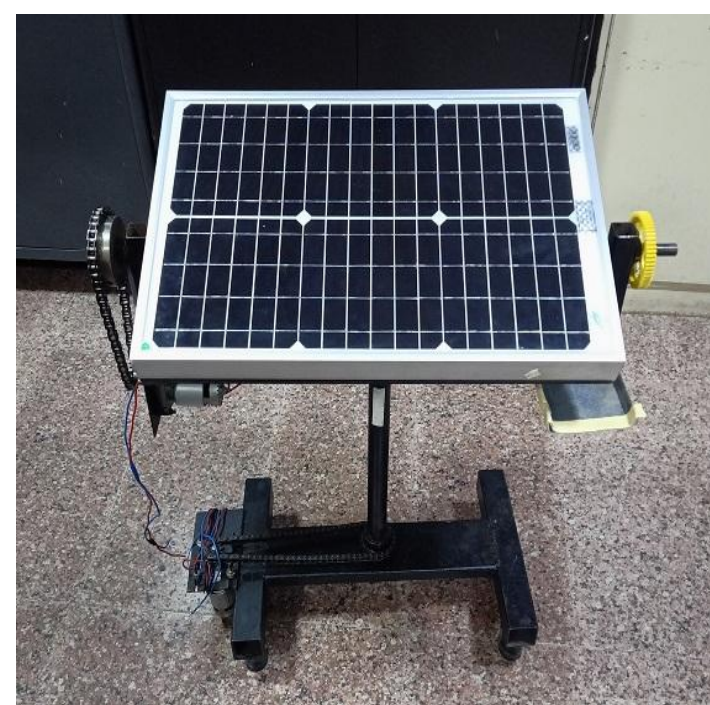

Fig. 5. Physical Model

\section{A. Solar Panel}

Solar panels receive light from sun as a source of energy to produce power. A photovoltaic (PV) module is a bundled, associated get together of regularly $6 \times 10$ photovoltaic solar cells. Photovoltaic modules consist of the photovoltaic array of a photovoltaic system that produces and supplies solar power in commercial and house hold applications.

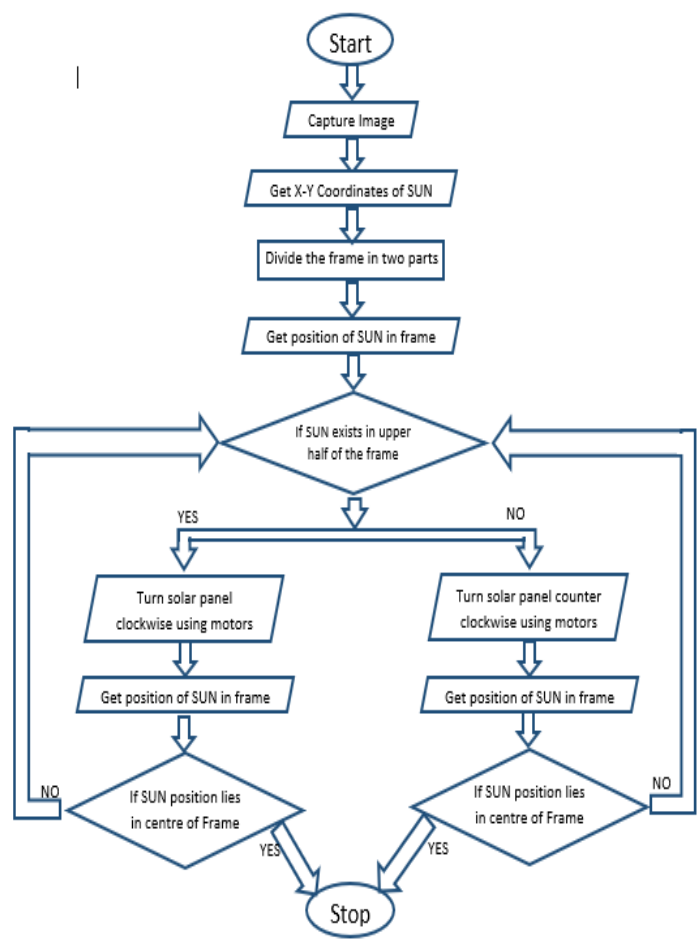

Fig. 6. Motion Work Flow Process

\section{B. DC Motor}

DC Motor used in this system has maximum $100 \mathrm{RPM}$ at $12 \mathrm{~V}$ constant DC supply. The DC motor is controlled using L298N motor driver module by a reprogrammable microcontroller. It is having a Torque of $12 \mathrm{Kg}-\mathrm{cm}$. It works on the principle of Fleming's Left-hand rule.

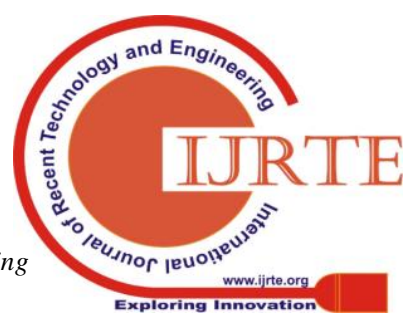


The physical model of the system is shown in fig. 5. An image of sun is captured and the centre point coordinates of sun are obtained. A micro controller is connected that is Arduino UNO to the raspberry pi. A solar panel is divided into two sections, that is upper half and lower half. When the system is placed outside, a webcam is utilized to capture image of the sun, Arduino UNO (micro controller) gets the position of sun in frame. Check the position of sun in frame whether it is in upper half or lower half of the frame. Then Arduino UNO micro controller sends signal to the motor driver to turn the solar panel to the required position. Then motor driver controls the DC motor motion to acquire the adequate position. Arduino again, checks the position of sun and sends signal to the motor driver, this keeps on going. When there is no sun or high dense region in the image captured then no operation will take place. Entire work flow process is shown in fig. 6 .

\section{CONCLUSION}

In this paper, we propose an economical dual-axis solar tracking system which is not dependent on the initial settings or geographical location. The developed prototype of solar tracking system could track the Sun with an accuracy of $\pm 1.5^{\circ}$ and constantly maintain the solar panel perpendicular to the Sun's radiation direction. A DC motor was used in this system to make the system economical and reduce the need of complicated drives while maintaining system accuracy. The overall efficiency of the proposed system's power output is increased by a factor of $6.87 \%$ from a stationary solar panel from $1 \mathrm{pm}$ to $6 \mathrm{pm}$ (IST). Hence, obtaining maximum efficiency throughout the day.

\section{REFERENCES}

1. M. Abdollahpour, M. R. Golzarian, A. Rohani, and H. Abootorabi Zarchi, "Development of a machine vision dualaxis solar tracking system," Solar Energy, vol. 169, no. July 2017, pp. 136-143, 2018.

2. F. Hénault, M. Coquand, P. H. Defieux, and C. Caliot, "Sun backward gazing method with multiple cameras for characterizing solar concentrators," Solar Energy, vol. 166, no. January, pp. 103-114, 2018.

3. Y. Yao, Y. Hu, S. Gao, G. Yang, and J. Du, "A multipurpose dual-axis solar tracker with two tracking strategies," Renewable Energy, vol. 72, pp. 88-98, 2014.

4. T. P. Chang, "Performance study on the east-west oriented single-axis tracked panel," Energy, vol. 34, no. 10, pp. 1530$1538,2009$.

5. M. C. Ho et al., "Design and Construction of Prototype Mobile Sun-Tracking System for Concentrator Photovoltaic System," Energy Procedia, vol. 142, pp. 736-742, 2017.

6. Z. W. Gai, S. Q. Gao, and J. Long, "Design of Automatic Sunlight Tracking Solar Panel System Based on Single Chip Microcomputer," Advanced Material Resource, vol. 834-836, pp. 1128-1131, 2013.

7. F. Sallaberry, R. Pujol-Nadal, M. Larcher, and M. H. Rittmann-Frank, "Direct tracking error characterization on a single-axis solar tracker," Energy Conversion Management, vol. 105, pp. 1281-1290, 2015.

8. B. J. Huang, W. L. Ding, and Y. C. Huang, "Long-term field test of solar PV power generation using one-axis 3-position sun tracker," Solar Energy, vol. 85, no. 9, pp. 1935-1944, 2011.

9. N. Mohammad and T. Karim, "Design and Implementation of Hybrid Automatic Solar-Tracking System," J. Solar Energy Engineering, vol. 135, no. 1, p. 011013, 2012.
10. L. Barker, M. Neber, and H. Lee, "Design of a low-profile two-axis solar tracker," Solar Energy, vol. 97, pp. 569-576, 2013.

11. Y. Yao, Y. Hu, S. Gao, G. Yang, and J. Du, "A multipurpose dual-axis solar tracker with two tracking strategies," Reneable Energy, vol. 72, pp. 88-98, 2014.

12. C. Sungur, "Multi-axes sun-tracking system with PLC control for photovoltaic panels in Turkey," Renewable Energy, vol. 34, no. 4, pp. 1119-1125, 2009.

13. G. Li, R. Tang, and H. Zhong, "Optical performance of horizontal single-axis tracked solar panels," Energy Procedia, vol. 16, no. PART C, pp. 1744-1752, 2011.

14. T. Tomson, "Discrete two-positional tracking of solar collectors," Renewable Energy, vol. 33, no. 3, pp. 400-405, 2008.

15. Sanjanna, Y. Bevish Jinila (2015), “An Approach on Automated Rescue System with Intelligent Traffic Lights for Emergency Services", International Conference on Innovations in Information, Embedded and Communication Systems (ICIIECS 2015), pp. 1-5, IEEE

16. Anu, V. Maria, D. Sarikha, G. Sai Keerthy, and J. Jabez. "An RFID based system for bus location tracking and display." In International Confernce on Innovation Information in Computing Technologies, pp. 1-6. IEEE, 2015.

17. Jeba Blessingson, Y.Bevish Jinila (2010), "Multi Utility / Tracing Kit for vehicles using RFID Technology", Recent Advances in Space Technology Services and Climate Change -2010, PP. 273 - 276, IEEE 